\title{
Rendimiento productivo del cerdo criollo alto andino en un sistema de producción
} intensiva

Productive performance of the high andean creole pig in an intensive production system Hilario Noberto Pujada Abad', Rufino Máximo Maguiña Maza', Dionisio Belisario Luis Olivas², Félix Esteban Airahuacho Bautista

\section{RESUMEN}

Objetivo: Determinar características productivas y reproductivas del cerdo criollo altoandino en un módulo intensivo. Métodos: Se utilizó doce hembras y dos machos adquiridos a pobladores de los distritos de Santa Leonor y Checras, provincia de Huaura, Región Lima - Provincias. Se realizó el análisis estadístico descriptivo para las variables productivas y reproductivas. Resultados: se obtuvo peso de camada e individual al nacimiento de 9,12 y $1,13 \mathrm{~kg}$, respectivamente, 0,90 lechones nacidos con peso menor de un $\mathrm{kg}$, peso de camada e individual de destete a los 35 días de 39,65 y 5,28, respectivamente. Las reproductoras tuvieron una gestación promedio de 113,8 días y lechones nacidos vivos por parto de 8,05 individuos. Conclusiones: Los rendimientos productivos y reproductivos de cerdos criollos en sistema intensivo del presente estudio se muestran superiores comparados con rendimientos de cerdos criollos latinoamericanos en sistema de crianza extensivo, pero inferiores a rendimientos alcanzados en cerdos genéticamente mejorados.

Palabras clave: Cerdo criollo, índices zoométricos, fanerópticas

\section{ABSTRACT}

Objective: To determine the productive and reproductive characteristics of the high Andean creole pig in an intensive module. Methods: Twelve females and two males acquired from residents of the districts of Santa Leonor and Checras, province of Huaura, Lima Region - Provinces. The descriptive statistical analysis was carried out for the productive and reproductive variables. Results: litter weight and individual weight at birth of 9.12 and $1.13 \mathrm{~kg}$, respectively, were obtained, 0.90 piglets born with a weight less than one $\mathrm{kg}$, litter weight and weaning individual at 35 days of 39.65 and 5.28, respectively. The breeders had an average gestation of 113.8 days and piglets born alive by birth of 8.05 individuals. Conclusions: The productive and reproductive yields of Creole pigs in the intensive system of the present study are superior compared to yields of Latin American creole pigs in extensive breeding system, but lower than yields achieved in genetically improved pigs.

Keywords: Creole pig, zoomometric, phaneroptic indices

\footnotetext{
${ }^{1}$ Escuela de Ingeniería Zootécnica. Universidad Nacional José Faustino Sánchez Carrión. Huacho, Lima, Perú.

${ }^{2}$ Escuela de Ingeniería Agronómica. Universidad Nacional José Faustino Sánchez Carrión. Huacho, Lima, Perú.
} 


\section{INTRODUCCIÓN}

El cerdo denominado "criollo", que habita Latinoamérica, tienen su origen en los cerdos de raza Ibérica, que llegaron a América en 1493, en el segundo viaje de Colón a la isla La Española (Pinheiro, 1976). En general, el cerdo criollo es un animal tipo graso, de tamaño mediano y de capa negra predominante, aunque presenta diferentes coloraciones en su capa por el cruzamiento con otras razas a lo largo de los años (Benítez y Sánchez, 2001). Según el Ministerio de Agricultura y Riego (2012) los cerdos criollos representan el $67,2 \%$ de la ganadería porcina, distribuido su crianza en $38,8 \%, 86,8 \%$ y $79,2 \%$ en la Costa, Sierra y Selva, respectivamente.

Los cerdos criollos son considerados rústicos por naturaleza debido a que toleran elevadas temperaturas, piel resistente a la fotosensibilidad, adaptados a terrenos agrestes, resistente a ectoparásitos y capacidad digestiva a alimentos vegetales de baja calidad (Scarpa, Drucker, Anderson y Ferres-Ehuan, 2003). Aparentemente, la alta rusticidad del cerdo criollo limitaría mostrar todo su potencial genético productivo. Comparado con los rendimientos productivos de razas mejoradas de regímenes intensivos, los cerdos criollos muestran rendimientos productivos inferiores. Sin embargo, bajo las condiciones habituales de manejo, alimentación y sanidad en el que se encuentran, no requieren de insumos de calidad (Santana, 1999; Gómez, 2004). Según Tapia (2009), el cerdo criollo latinoamericano presenta baja velocidad de crecimiento, deficiente eficiencia alimenticia, menor porcentaje de tejido magro y pequeño tamaño de camada, entre otros.

En ese escenario, el cerdo criollo representa una alternativa en la generación de recursos económicos, sobre todo en las zonas altoandinas en las que se debe aprovechar al máximo el uso de los recursos disponibles. Asimismo, su explotación permite mantener material genético adaptado a esas condiciones ambientales. Por lo que la preservación genética de animales de granja y de fauna silvestre ha sido reconocida por la FAO como un componente del Programa Mundial de recuperación de la biodiversidad biológica (Zurkowski, 1997). El material genético animal constituye la materia prima que permite establecer sistemas de producción en función a las demandas de los consumidores y las exigencias de mercados cada vez más competitivos. Sin embargo, para manejar y aprovechar adecuadamente los recursos genéticos animales tienen que ser caracterizados y conservados a fin de hacerlos accesibles en cualquier momento (Alarcón y González, 1996).

Como toda especie animal, el cerdo criollo tiene potencial genético que podría manifestarse si las condiciones ambientales adecuadas le son proporcionadas. Por ello, la presente investigación evaluó el comportamiento productivo del cerdo criollo altoandino en un sistema de crianza intensivo.

\section{MATERIALES Y METODOS}

La investigación fue realizada en la granja experimental de cerdos criollos de la Universidad Nacional José Faustino Sánchez Carrión, con coordenadas $11^{\circ} 07^{\prime \prime} 29$ LS y $77^{\circ} 36^{\prime \prime} 35^{\prime \prime}$ LO, entre enero del 2014 y diciembre del 2016. Los animales fueron alimentados con raciones de niveles nutricionales recomendados para cerdos ibéricos según las normas FEDNA (2013). El pie de cría inicial utilizado en el estudio fue de 12 hembras y 2 machos de 5 meses de edad, y de $30 \mathrm{~kg}$ de peso vivo promedio. Las características productivas y reproductivas medidas en un intervalo de tres partos fueron el número de lechones nacidos, peso de camada e individual al nacimiento, peso de camada e individual al destete, días en gestación y numero de lechones nacidos vivos.

El ritmo de crecimiento fue determinado semanalmente en cada uno de los lechones nacidos, desde el día de nacido hasta los 120 días de edad. Se estableció la relación entre peso y edad del animal, a través de un modelo de regresión lineal. Para evaluar las diferencias entre características productivas y reproductivas en relación con el número de partos, se utilizó el ANOVA y cuando se evidenció diferencias significativas, se aplicó la Prueba de Tukey con un nivel del $5 \%$ de probabilidad. 


\section{RESULTADOS}

Los parámetros productivos medidos no fueron afectados por el número de partos de la marrana (Tabla 1). Aunque no se encontró diferencias estadísticas, marranas del tercer parto presentaron un lechón nacido por parto con peso menor a un $\mathrm{kg}$. Asimismo, el peso de camada e individual al nacimiento fue numéricamente superior en marranas del tercer parto, pero el peso individual por lechón en la etapa de destete fue inferior comparado con pesos de lechones del primer y segundo parto.

El promedio de granja observado para los parámetros productivos fue de $0,90 \pm 1,16$ lechones nacidos con pesos menor de $1 \mathrm{~kg}$, $9,12 \pm 2,16 \mathrm{~kg}$ de peso de carnada al nacimiento, $1,133 \pm 0,10 \mathrm{~kg}$ de peso del lechón al nacimiento, 39,65 $\pm 13,23 \mathrm{~kg}$ de peso de carnada al destete, $5,283 \pm 1,18 \mathrm{~kg}$ de peso del lechón al destete, y 34,95 $\pm 2,04$ días en lactación (Tabla 1).

Tabla 1. Características productivas de cerdos criollos en crianza intensiva.

\begin{tabular}{|c|c|c|c|c|c|c|}
\hline $\begin{array}{l}\mathbf{N}^{\circ} \\
\text { Partos }\end{array}$ & $\begin{array}{l}\text { Lechones } \\
\text { nacidos peso } \\
\text { menor un kg } \\
(\mathrm{L}<01 \mathrm{Kg}) \text {, }\end{array}$ & $\begin{array}{l}\text { Peso camada } \\
\text { Nacimiento } \\
\text { (PCN; kg) }\end{array}$ & $\begin{array}{l}\text { Peso lechón } \\
\text { Nacimiento } \\
\text { (PLN; kg) }\end{array}$ & $\begin{array}{l}\text { Peso } \\
\text { camada } \\
\text { Destete } \\
\text { (PCD; kg) }\end{array}$ & $\begin{array}{l}\text { Peso } \\
\text { lechón } \\
\text { Destetado } \\
\text { (PLD; kg) }\end{array}$ & $\begin{array}{l}\text { Días } \\
\text { lactación }\end{array}$ \\
\hline 1 & $0,89^{a} \pm 1.0$ & $8,22^{\mathrm{a}} \pm 2.2$ & $1,12^{a} \pm 0,1$ & $34,63^{a} \pm 8,0$ & $5,20^{\mathrm{a}} \pm 1,4$ & $34,7 \mathrm{a} \pm 1,3$ \\
\hline 2 & $0,86^{a} \pm 0.9$ & $9,33^{a} \pm 1.7$ & $1,11^{\mathrm{a}} \pm 0.1$ & $46,15^{\mathrm{a}} \pm 17,1$ & $5,70^{a} \pm 1,1$ & $35,7^{a} \pm 12,6$ \\
\hline 3 & $1,00^{a} \pm 2,0$ & $10,77^{\mathrm{a}} \pm 2,1$ & $1,21^{a} \pm 0,2$ & $39,58^{a} \pm 13,5$ & $4,38^{a} \pm 0,8$ & $34,2^{a} \pm 1,5$ \\
\hline Promedio & $0,90 \pm 1,2$ & $9,12+2,2$ & $1,13 \pm 0,1$ & $39,65 \pm 13,2$ & $5,28 \pm 1,2$ & $34,95 \pm 2,0$ \\
\hline
\end{tabular}

${ }^{\text {abc }}$ letras iguales son similares estadísticamente $(p<0,05)$.

El ritmo de crecimiento de los cerdos evaluados se ajusta al modelo de regresión polinomial de segundo orden. A la vista del resultado analítico del modelo para hembras es bueno ya que el valor $R^{2}=0,805$ es cercano a 1 , es decir, el $80,5 \%$ de la variabilidad de la variable peso vivo a su promedio es explicado por el modelo de regresión ajustado. Para machos, la asociación es fuerte $\left(R^{2}=0,978\right)$, observándose que el modelo polinomial es adecuado para describir la relación que existe entre estas variables.
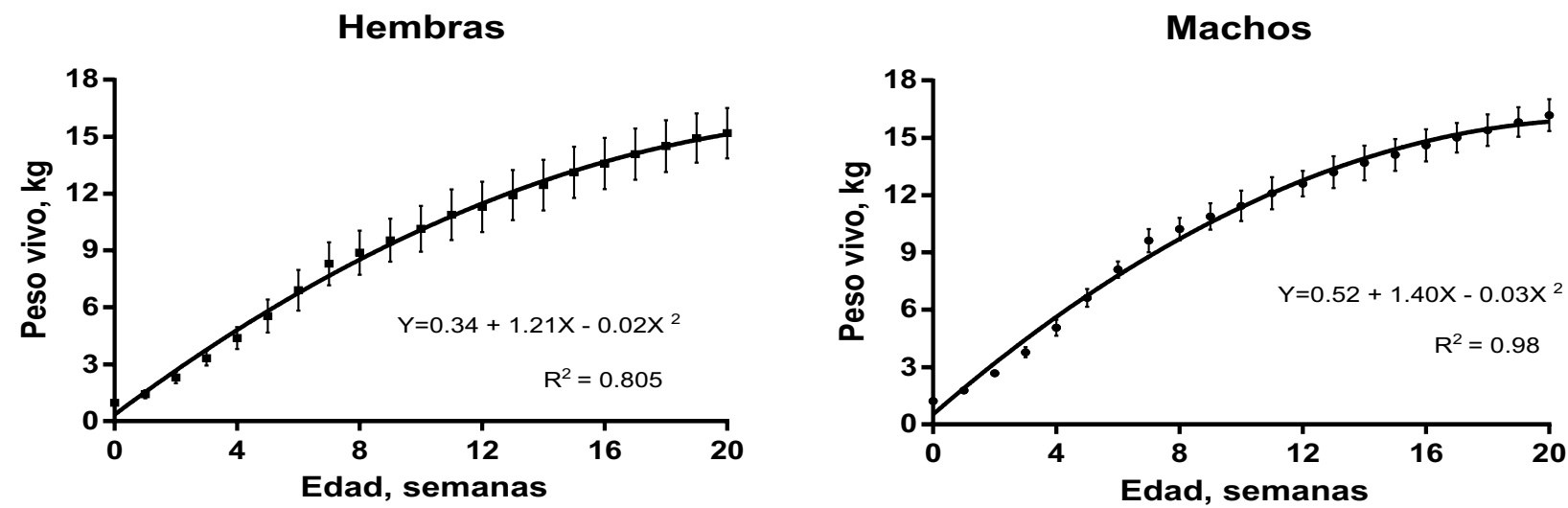

Figura 1. Ritmo de crecimiento de los cerdos criollos bajo condiciones de crianza intensiva. 
Los días de gestación de las marranas y el número de lechones nacidos vivos por parto no mostro diferencias estadísticas entre el número de partos (Tabla 2). El promedio general observado para días en gestación fue de $113,8 \pm 1,2$; en tanto que, para lechones nacidos vivos fue de $8,05 \pm 2,0$.

Tabla 2. Rendimiento reproductivo de cerdos criollos en crianza intensiva.

\begin{tabular}{lll}
\hline $\mathbf{N}^{\circ}$ de partos & Días en gestación & Lechones nacidos vivos $\left(\mathbf{N}^{\circ}\right)$ \\
\hline 1 & $113,7^{\mathrm{a}} \pm 1,1$ & $7,4^{\mathrm{a}} \pm 2,3$ \\
2 & $114,0^{\mathrm{a}} \pm 1,0$ & $8,4^{\mathrm{a}} \pm 1,6$ \\
3 & $113,5^{\mathrm{a}} \pm 1,7$ & $8,8^{\mathrm{a}} \pm 1,7$ \\
\hline Promedio granja (4) & $113,8 \pm 1,2$ & $8,05 \pm 2,0$ \\
\hline
\end{tabular}

${ }^{a b c}$ letras iguales son similares estadísticamente $(p<0.05)$.

\section{DISCUSIÓN}

En el presente estudio, el peso de lechones nacidos con menos de un $\mathrm{kg}$ de peso vivo $(\mathrm{L}<01 \mathrm{Kg})$ fue de 0,90 lechones por parto. El peso de lechones al nacimiento con peso inferior a un $\mathrm{kg}$ de peso vivo estaría influenciado por la condición corporal de la marrana. Palomo (2014) menciona que la condición corporal de la hembra garantiza la longevidad, prolificidad y fertilidad que da lugar a camadas homogéneas. Sin embargo, las hembras de estudio en nuestra granja, con un programa de alimentación con alimento balanceado, mostraban condiciones corporales adecuadas.

El peso promedio de camada al nacimiento del presente estudio fue superior a otros índices reportados en otros estudios. En cerdos criollos tipo Zungo se reportó peso de camada de 7,66 $\mathrm{kg}$ (Cabeza, 1976), 5,66 kg en cerdos nativos de Pernambuco (Rodrígues, 2014) y $5,39 \mathrm{~kg}$ en cerdo criollo Pelón Mexicano (Lemus y Alonso, 2006). Sin embargo, fueron inferiores a los obtenidos por Vadell (2008) en cerdos criollos de Pampa-Rocha $(11,46$ a $12,19 \mathrm{~kg}$ de peso de camada al nacimiento) y por PIC (2011) con líneas genéticas mejoradas $(19,37 \mathrm{~kg}$ peso promedio camada), confirmando trabajos previos que indican que los cerdos criollos no son prolíficos (Castro, 1981). Posiblemente el peso de camada esté relacionado con la restricción del espacio uterino disponible durante la fase de crecimiento del útero, su vascularización y los receptores uterinos (Palomo, 2014).

El peso de lechón al nacimiento fue de $1,13 \pm 0,1 \mathrm{~kg}$ por lechón recién nacido (Tabla 1). Cabeza (1976). Mota, Ramírez, Alonso y García (2001) y Rodríguez (2014) reportaron peso de lechón al nacimiento de 0,$970 ; 0,950 ; 0,943 \mathrm{~kg}$, respectivamente, valores inferiores a los hallados en el presente estudio. Sin embargo, los resultados obtenidos en esta investigación fueron ligeramente inferiores a los reportados por Vadell (2008) que obtuvo de 1,18 a 1,33 kg en lechones criollos de las Pampas-Rocha, así como el peso de nacimiento promedio $1,49 \mathrm{~kg}$ de líneas genéticas mejoradas (PIC, 2011). El tamaño de carnada influencia en el peso del lechón al nacimiento por tener un mayor espacio en el útero, aumentando las posibilidades de una alimentación a plenitud durante la gestación, y repercutiendo sobre la ganancia de peso durante esta etapa 
(Canario, Lundgren, Haandlykken y Rydmer, 2010).

El peso de carnada al destete fue de $39,6 \pm 13,2 \mathrm{~kg}$ (Tabla 1). Resultados inferiores como 23,2 kg fueron reportados por Benítez y Sánchez (2001) en cerdos criollos mexicanos, mientras Lemus y Alonso (2006) reportan pesos de 18,9 kg en cerdos criollos de Ecuador. Resultados superiores como $72 \mathrm{~kg}$ de peso camada a los 21 días han sido reportados por PIC (2011); mientras Carr (2004) propone 7,0 kg para el porcino precoz de capa blanca a los 28 días de edad. Posiblemente, la nutrición de la cerda lactante tiene un efecto sobre la composición de la leche y sobre los resultados del crecimiento de peso vivo en los lactantes, así como el potencial genético magro, que el cerdo criollo no lo tiene (Foxcroft et al., 2006).

El peso del lechón al destete fue de 5,28 $1,2 \mathrm{~kg}$ (Tabla 1). Resultados como $7,8 \mathrm{~kg}$ en cerdo colombiano, $6,8 \mathrm{~kg}$ en cerdo ecuatoriano y 5,3 en cerdos mexicanos han sido reportados por Salamanca, Arias y Vélez (2015); Pérez, Reyes, Velázquez, Delgado y Barba (2002) y Lemus y Alonso (2006) respectivamente. PIC (2011) marca como objetivo para su línea materna Camborough un peso vivo de seis $\mathrm{kg}$ a los 21 días de vida, mientras Carr (2004) propone $7,0 \mathrm{~kg}$ para el porcino precoz de capa blanca a los 28 días de edad. En cerdos criollos, la producción de leche de la cerda posiblemente sea un recurso limitante debido a que el consumo diario controlado de acuerdo con el crecimiento de las crías y la producción de leche de la cerda recién alcanza un nivel constante después del $8^{\circ}$ al $10^{\circ}$ día (Bouwman, Bergsma, Duijvesteijn y Bijma, 2010). Otra fuente potencial que altera la interacción es el establecimiento en el orden del pezón. Durante las primeras horas después del nacimiento los lechones empujan y muerden a los otros para capturar un pezón, pero la competencia se reduce tan pronto como el orden del pezón se ha establecido (Bouwman et al., 2010).

Los días de lactación fueron de $34.95 \pm 2,0$ días (Tabla 1). El tiempo de lactancia empleado en diferentes países para el cerdo criollo es variado, como 42 días (Salamanca et al., 2015) y 39 días (Lemus y Alonso, 2006). Asimismo, Monteverde (2001) en lechones criollos tipo Pampas en Uruguay empleó 21 días de lactación. La línea genética comercial PIC, tiene como objetivo para su línea materna Camborough periodos de lactancia de 21 días (PIC, 2011). No es conveniente emplear periodos de lactancia prolongadas, debido a que la cerda en lactación tiene una incapacidad de ingerir todo el alimento necesario para mantener su gran producción láctea. Por este motivo las cerdas necesitan movilizar parte de sus reservas corporales para cubrir sus necesidades y si la pérdida es excesiva (especialmente en primíparas), se puede esperar un balance energético y proteico negativo durante la lactación. Esto conlleva a afectar el desarrollo, restablecimiento y selección del grupo de folículos pre-ovulatorios y un cambio gradual en el número de folículos a las categorías de medio y gran tamaño, y el decrecimiento del porcentaje de folículos atrésicos (Kunavongrit, 1982).

El ritmo de crecimiento determinado por el modelo de regresión polinomial de segundo grado muestra una fuerte asociación $\left(R^{2}=80,5 \%\right.$ y $R^{2}=97,8 \%$, para hembras y machos, respectivamente). Estos resultados son similares a los mostrados por la línea genética de engorde PIC con $R^{2}$ de 94,4 \% (PIC, 2011).

El tiempo de gestación (días), fue de 113,8 \pm 1,2 días (Tabla 2), similar a lo reportado en Ecuador y México en este mismo tipo de animal (Lemus y Alonso 2006; Hafez y Hafez, 2002), asimismo son similares a las razas puras (Lancho de León, Detena y Porras, 1975) y cerdos 
mejorados (PIC, 2011), lo que demuestra que es una característica propia de especie Sus scrofa (Johansson y Rendel, 1972).

El número de lechones nacidos vivos fue de 8,05 $\pm 2,0$ lechones por parto (Tabla 2). Resultados diferentes como 7,4 lechones colombianos, 7,25 lechones cubanos y 5,6 lechones mexicanos, han sido reportados por Salamanca et al. (2015); Pérez et al., (2002); Lemus y Alonso (2006) respectivamente. Vadell (2008) y Monteverde (2001) en cerdos criollos Pampa-Rocha reportan 9,1 a 9,9 lechones vivos por parto. Cerdas de líneas mejoradas alcanzan en promedio 13 lechones nacidos vivos por parto (PIC, 2011). El número de lechones nacidos vivos, promedios obtenidos en la investigación, posiblemente sea por la alimentación reflejada en el peso del apareamiento, y no por la tasa de ovulación, supervivencia embrionaria y capacidad uterina, que responden a la selección genética (Hafez y Hafez, 2002; Foxcroft et al., 2006).

En condiciones altoandinas, por lo general, es aceptado que las razas criollas alcanzan mejores parámetros productivos que las razas foráneas introducidas (Cardona, Londoño y Echeverri, 2017). En el presente estudio, si bien los rendimientos productivos de los cerdos criollos se muestran inferiores a los altos rendimientos reportados para cerdos mejorados, los resultados se muestran superiores a los cerdos criollos de otros países sudamericanos explotados en sistemas extensivos. Estas mejoras estarian relacionadas debido a que el presente estudio se desarrolló en instalaciones y alimentación adecuadas.

\section{REVISIÓN BIBLIOGRÁFICA}

Alarcón, E., \& Gonzales, E. (1996). Utilización sostenible y conservación de los recursos genéticos animales en las Américas mediante la cooperación técnica interinstitucional. In Memorias $3 e r$ Congreso Iberoamericano de razas Autóctonas y Criollas (pp. 5-25).

Benítez, W., \& Sánchez, D.M. (2001). Los cerdos criollos en América Latina. En: FAO (ed.) Los cerdos locales en los sistemas tradicionales de producción. FAO Producción y Sanidad Animal, 1335.

Bouwman, A. C., Bergsma, R., Duijvesteijn, N., \& Bijma, P. (2010). Maternal and social genetic effects on average daily gain of piglets from birth until weaning. Journal of animal Science, 88(9), 2883-2892.

Cabeza, V.M. (1976). Estudio comparativo de la raza nativa de cerdo Zungo con razas mejoradas (Tesis de Maestría). Universidad Nacional de Colombia, Bogotá, Colombia.

Canario, L., Lundgren, H., Haandlykken, M., \& Rydhmer, L. (2010). Genetics of growth in piglets and the association with homogeneity of body weight within litters. Journal of animal Science, 88(4), 1240-1247.

Cardona, D., Londoño, M., \& Echeverri, J. J. (2017). Evaluación comparativa de parámetros productivos en diferentes cruces de ganado Blanco-Orejinegro con Holstein. Corpoica Ciencia y Tecnología Agropecuaria, 18(3), 513527.

Carr, J. (2004). Manual técnico estándares de la producción porcina. Zaragoza, España.

Castro, G.E.M. (1981). Importancia que ejercen algunos factores ambientales $y$ el efecto del semental sobre el tamaño y peso de la carnada al nacimiento y al destete en el Cerdo Pelón Mexicano (Tesis de pregrado). Universidad Nacional Autónoma de México. México, D.F.

Fundación Española para el Desarrollo de la Nutrición Animal (FEDNA). (2013). 
Necesidades nutricionales para ganado porcino. Madrid, España. Disponible en:

http://www.fundacionfedna.org/sites/de fault/files/Normas\%2OPORCINO_2013rev 2.pdf

Foxcroft, G.R, Dixon, W.T., Novak, S., Putman, C.T., Town, S.C. \& Vinsky, M. D. A. (2006). The biological basis for prenatal programming of postnatal performance in pigs. Journal Animal Science, 84, 105-112.

Gómez, M. (2004). Situación actual y descripción del porcino criollo peruano. En J.V. Delgado (Ed.), Biodiversidad Porcina Iberoamérica (pp. 191-200). Córdova, España: Universidad de Córdova.

Hafez, E., \& Hafez, B. (2002). Reproducción e Inseminación artificial en animales. México, México: Mc Graw Hill Interamericana.

Johansson, I., \& Rendel, J. (1972). Genética y mejora animal. Zaragoza, España: Ed. Acribia.

Kunavongrit, A. (julio-agosto de 1997). Aspectos Nutricionales de la Cerda Lactante. Revista Anaporc (169), 14.

Lancho de León. G., Detena, A., \& Porras, C. (1975). Variación racial del periodo de gestación en ganado porcino. Archivos de Zootecnia, 24(95-96), 245-250.

Lemus, C. \& Alonso, M.L. (2006). El cerdo Pelón Mexicano y otros cerdos criollos. 2005. Revista Computadorizada de Producción Porcina, 13(1), 251.

Ministerio de Agricultura \& Riego (MINAGRI) (2012). IV Censo Nacional Agropecuario.

Monteverde, S. (2001). Producción de leche de cerdas criollas Pampas y Duroc en un sistema a campo (Tesis de pregrado). Universidad de la República, Montevideo, Uruguay.
Mota, D., Ramírez, R., Alonso, M., \& García, A. (2001). Indicadores productivos y reproductivos en regiones porcícolas marginadas de Zapotitlán, Distrito Federal. Sociedades rurales, producción y medio ambiente, 2(2), 4.

Palomo, A.Y. (noviembre-diciembre de 2014). Factores nutricionales ligados al peso del lechón al nacimiento. Mundo Ganadero, (261), 34-38.

Pérez, E., Reyes, L., Velázquez, R., Delgado, B., Barba, C. (2002). Comportamiento de la descendencia de cerdos criollos cubanos. Archivos de Zootecnia, 51, 377-380.

Pig Improvement Company (PIC). (2011). Guía de manejo para las reproductoras Camborough. España.

Pinheiro, M. (1976). Los cerdos. Buenos Aires, Argentina: Hemisferio sur.

Ramos, D. (2008). Caracterización de la canal y la carne del cerdo criollo y de los productos cárnicos en el Departamento de Tumbes-Perú (Tesis de doctorado). Universidad de León, Nueva León, España.

Rodrígues T. (2014). Avaliação de diferentes grupos genéticos de suínos criados ao ar livre no semiárido pernambucano (Tese de doutorado). Universidade Federal Rural de Pernambuco, Pernambuco, Brasil.

Salamanca, C.A., Arias, L.J., \& Vélez, T.M. (2015). Efectos ambientales que influyen en el comportamiento productivo de cerdas criollas Sabaneras en la Granja El Picure, A rauca, Colombia. Actas Iberoamericanas de Conservación Animal, 6: 16-23.

Santana, I. (1999). Integración del cerdo criollo a los sistemas de explotación porcina. C. Gonzales, et al. (Eds.), Producción de cerdos (98-108). 
Maracay, Venezuela: Universidad Central de Venezuela.

Scarpa, R., Drucker, A., Anderson, S., \& Ferres, N. (2003). Valuing genetic resources in peasant economies: the case of 'hairless' creole pigs in Yucatan. Journal of Ecological Economics 45, 427-443.

Tapia, E.A. (2009). El cerdo Criollo en el Caribe y Latinoamérica. Sistema de Revisiones en Investigación Veterinaria de San Marcos.

Vadell, A. (2008). Una reseña corta sobre la raza criolla de cerdos Pampa-Rocha y su utilización en Uruguay. Revista Computarizada de Producción Porcina, 15(2), 105-112.

Zurkowski, M. (1997). Preservation of the genetic resources. Animal Science Papers and Reports, 15(3), 127130.

\section{Correo electrónico:}

befeen@hotmail.com

Revisión de pares:

Recibido: 04-10-2018

Aprobado: 21-12-2018 